Corrigendum

\title{
Corrigendum to "Development of dextrin-amphotericin B formulations for the treatment of Leishmaniasis" [Int. J. Biol. Macromol., 15 (2020) 276-288]
}

\author{
R. Silva-Carvalho ${ }^{\text {a }}$, J. Fidalgo a , K.R. Melo ${ }^{\text {b }}$, M.F. Queiroz ${ }^{\text {b }}$, S. Leal ${ }^{\text {a }}$, H.A. Rocha ${ }^{\text {b }}$, T. Cruz ${ }^{\text {c,d }}$, P. Parpot ${ }^{\text {a,e }}$, \\ A.M. Tomás ${ }^{\mathrm{c}, \mathrm{d}, \mathrm{f}}, \mathrm{M}$. Gama ${ }^{\mathrm{a}, *}$ \\ a CEB - Centre of Biological Engineering, University of Minho, Campus de Gualtar, 4710-057 Braga, Portugal \\ ${ }^{\mathrm{b}}$ Departamento de Bioquímica, Centro de Biociências, Universidade Federal do Rio Grande do Norte, Natal, RN, Brazil \\ c i3S - Instituto de Investigação e Inovação em Saúde, Universidade do Porto, Rua Alfredo Allen 208, 4200-135 Porto, Portugal \\ d IBMC - Instituto de Biologia Molecular e Celular, Universidade do Porto, Rua Alfredo Allen 208, 4200-135 Porto, Portugal \\ e Centre of Chemistry, University of Minho, Campus de Gualtar, 4710-057 Braga, Portugal \\ f ICBAS - Instituto de Ciências Biomédicas Abel Salazar, Universidade do Porto, Rua de Jorge Viterbo Ferreira 228, 4050-313 Porto, Portugal
}

The authors regret to inform that there are some inaccuracies in the above referred article. More specifically, in the end of the Results and Discussion section 3.6. Anti-Leishmania activity against axenic promastigote and intramacrophagic $L$. infantum amastigotes it is described the following "Dex-AmB nanocomplexes had an activity similar to the free-drug, despite the slightly higher $\mathrm{IC}_{50}$ values $-0.017 \pm 0.009$ and $0.023 \pm 0.006 \mu \mathrm{M}$ for Dex-AmB FD and Dex-AmB SD, respectively promoting a decrease in the amastigote parasite burden in the used concentration range. These results are consistent with the literature where reduced and unreduced AmB-AG conjugates were able to decrease the percentage of macrophages infected with $L$. infantum $\left(E_{50}\right.$ of
$0.035 \mathrm{mg} / \mathrm{mL}$ and $0.027 \mathrm{mg} / \mathrm{mL}$, respectively) [75]". In this sentence the units "mg/mL" are incorrect and the correct ones are " $\mu \mathrm{g} / \mathrm{mL}$ ". Moreover, the reference numbered with 75 should be "J. Golenser, et al., Efficacious treatment of experimental leishmaniasis with amphotericin Barabinogalactan water-soluble derivatives. Antimicrob Agents Chemother 43(9) (1999) 2209-14" instead of "R. Falk, et al. A novel injectable water-soluble amphotericin B-arabinogalactan conjugate. Antimicrob. Agents Chemother., 43 (8) (1999), pp. 1975-1981".

The authors would like to apologise for any inconvenience caused.

DOI of original article: https://doi.org/10.1016/j.ijbiomac.2020.03.019.

* Corresponding author at: Department of Biological Engineering, University of Minho, Campus de Gualtar, 4710-057 Braga, Portugal.

E-mail address: fmgama@deb.uminho.pt (M. Gama). 